\title{
Interference-free Detection of Caffeine in Complex Matrices Using Nanochannels Electrode Modified with Binary hydrophilic-hydrophobic PDMS
}

Yintao Lia, Zisheng Luo a,b,c, Gangfeng Lia, Tarun Belwala, Li Lia,b, Yanqun Xua,c, Bin $\mathrm{Su}^{\mathrm{d}}$, Xingyu Lin*a,b

a Zhejiang University, College of Biosystems Engineering and Food Science, Key Laboratory of Agro-Products Postharvest Handling of Ministry of Agriculture and Rural Affairs, Zhejiang Key Laboratory for Agri-Food Processing, Hangzhou, 310058, China

${ }^{\mathrm{b}}$ China National-Local Joint Engineering Laboratory of Intelligent Food Technology and Equipment, Zhejiang R\&D Center for Food Technology and Equipment, Hangzhou, 310058, China c Ningbo Research Institute, Zhejiang University, Ningbo, 315100, China

d Institute of Analytical Chemistry, Department of Chemistry, Zhejiang University, Hangzhou 310058, China

*Corresponding Author: Xingyu Lin (xingyu@zju.edu.cn) 
1. Plasma-triggered PDMS deposition

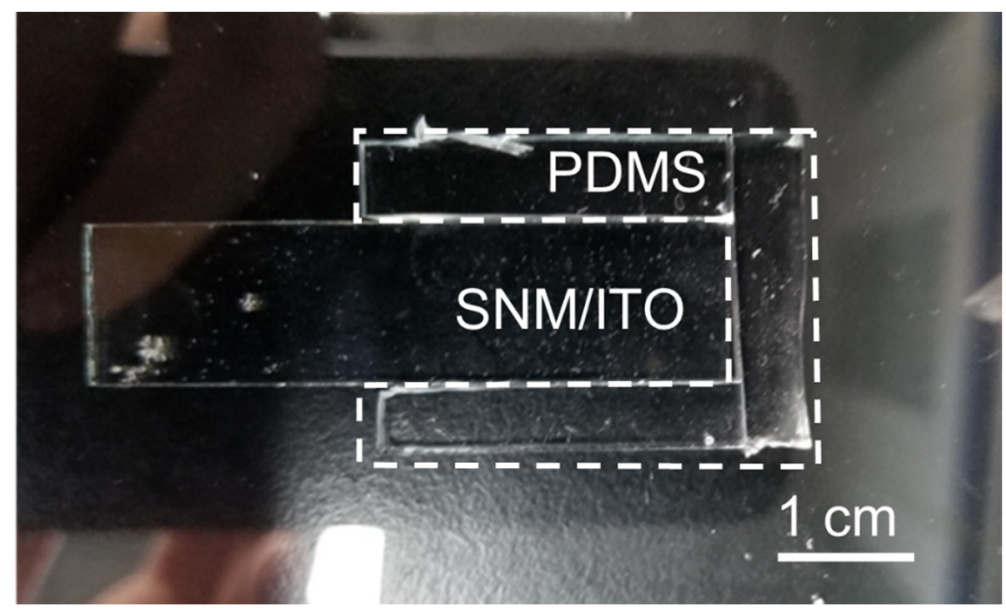

Fig S1. The photograph of electrode surrounded by the bulk PDMS.

2. Characterization of plasma triggered ultrafast PDMS deposition

(a)

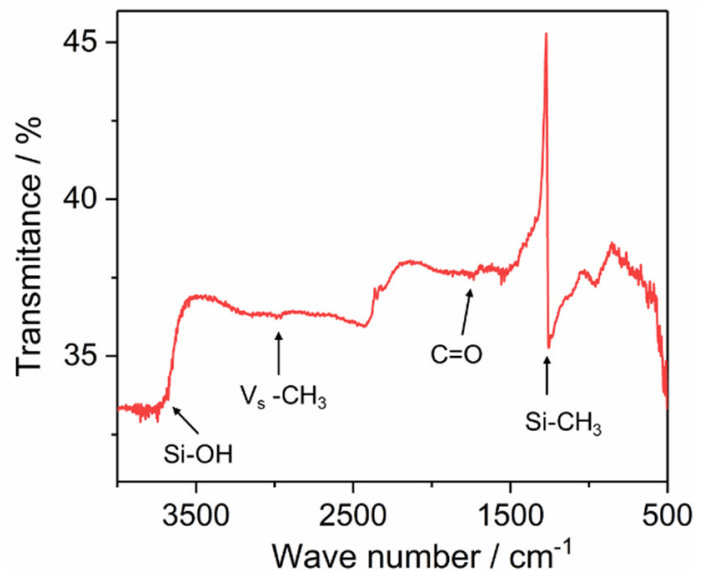

(b)

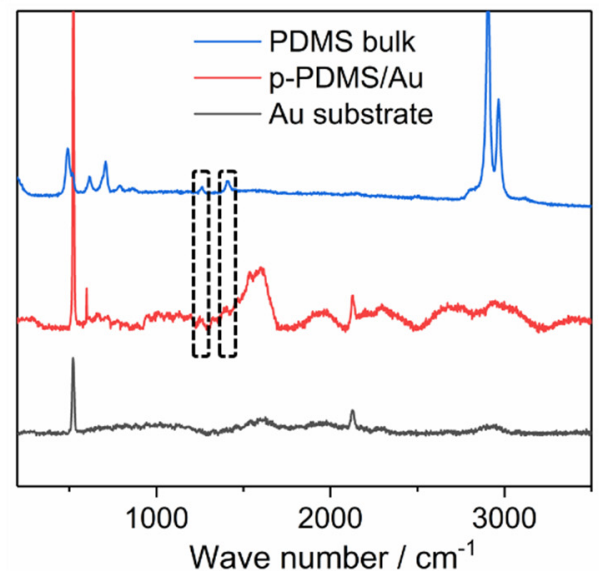

Fig. S2. (a) Grazing angle specular reflection FTIR spectra of p-PDMS/SNM. In this case, pPDMS/SNM/ITO was used as sample, while SNM/ITO was used as background. (b) Raman spectra of PDMS, Au substrate and PDMS coating substrate (power: 1.4MW, exposure time: $60 \mathrm{~s}$ ).

In order to improve the sensitivity, we used a grazing angle reflector in IR measurement. As shown in Fig. S2a, the peak around $3600 \mathrm{~cm}^{-1}$ should be derived from Si-OH stretching vibration. The peak around $1260 \mathrm{~cm}^{-1}$ and $1110 / 1000 \mathrm{~cm}^{-1}$ were derived from $\mathrm{Si}-\mathrm{CH}_{3}$ deformation vibration and Si-O-Si stretching vibration, respectively. A small peak also appeared around $1750 \mathrm{~cm}^{-1}$, which may come from the stretching vibration of carbonyl group generated after PDMS oxidation. In Raman spectrum, due to the ultra-thin deposited layer, Au substrate was used for surface enhanced Raman Scattering (SERS). Several different peaks appeared at p-PDMS deposited substrate, which were consistent with part of Raman signals of PDMS bulk, like the peaks around $1260 \mathrm{~cm}^{-1}$ and $1400 \mathrm{~cm}^{-}$ 1. Other signals that differed from the original PDMS may result from changes in groups of PDMS after oxidation. 
(a)

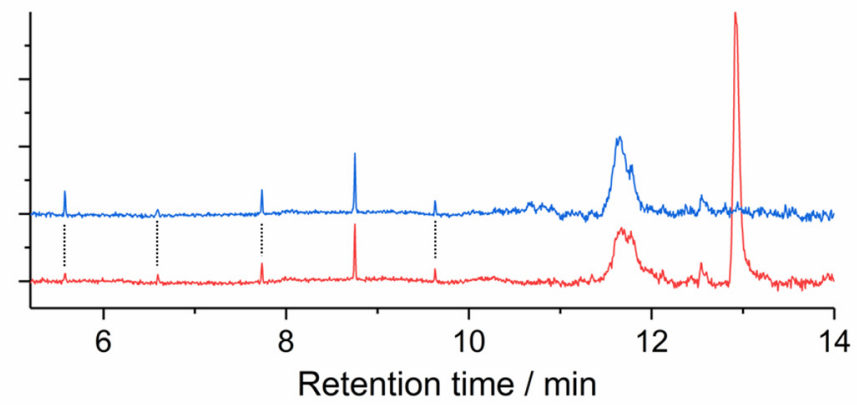

(b)

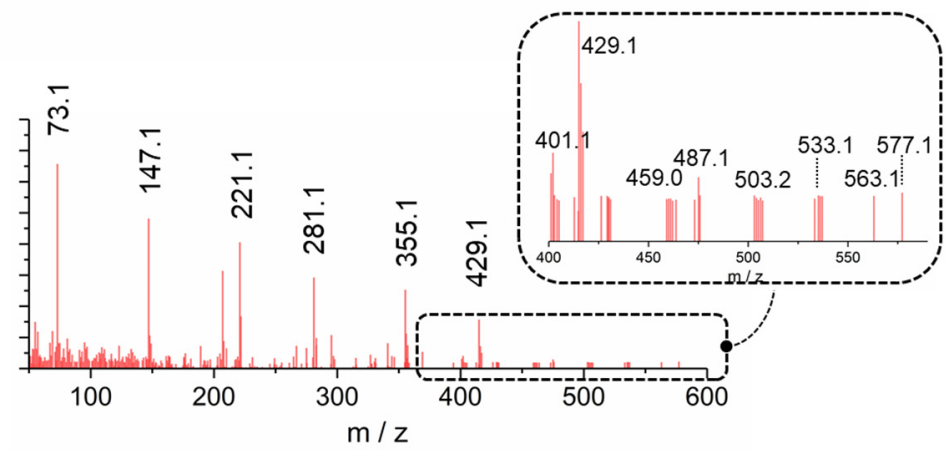

Fig S3. (a) Gas chromatogram with mass analysis. The blue data shows the GC-MS results of bulk PDMS, while the red data shows the results of deposited p-PDMS. (b) mass analysis of the components in p-PDMS which have a longer retention time (12.91 min).

To further support our view that the deposited p-PDMS indeed contain both the original PDMS oligomers and the oxidized ones, we compared the p-PDMS and the original PDMS oligomer using a GC-MS method. As shown in Fig. S3, for two types of PDMS, the peaks before 10min were highly coincident. ${ }^{1}$ This confirms that $\mathrm{p}$-PDMS deposited on the nanochannels does contain unoxidized PDMS oligomers similar to that of bulk PDMS. Also, more substances with longer retention time were found in p-PDMS. Generally, hydrocarbons would have a longer retention time after oxidation to oxygenated compounds with a higher boiling point. ${ }^{2}$ Besides, the mass spectral showed not only the characteristic fragments of siloxane but also several peaks differed by 44 or 28 , which may be attributed to the fragmentation of a carboxyl or carbonyl group. According to the previous studies, oxygen-containing groups such as carboxyl groups would appear in plasma-oxidized PDMS. ${ }^{3}$ Therefore, we suppose that p-PDMS obtained by plasma-triggered modification is a mixture containing both the oxidized hydrophilic PDMS oligomers and the original hydrophobic ones. This phenomenon may be caused by ultrafast deposition. During plasma irradiation, hydrophobic PDMS oligomers would come out from body material and deposited on the substrate. The PDMS oligomers on the nanochannel surface would become more hydrophilic due to the more exposure time to plasma irradiation, while oligomers inside the nanochannels would be relatively hydrophobic. 
3. Electrochemical characterization of p-PDMS/SNM/ITO

(a)

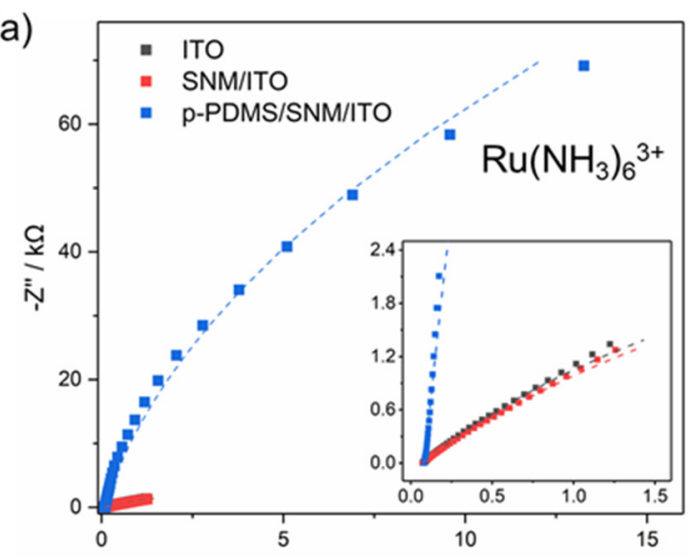

(c)

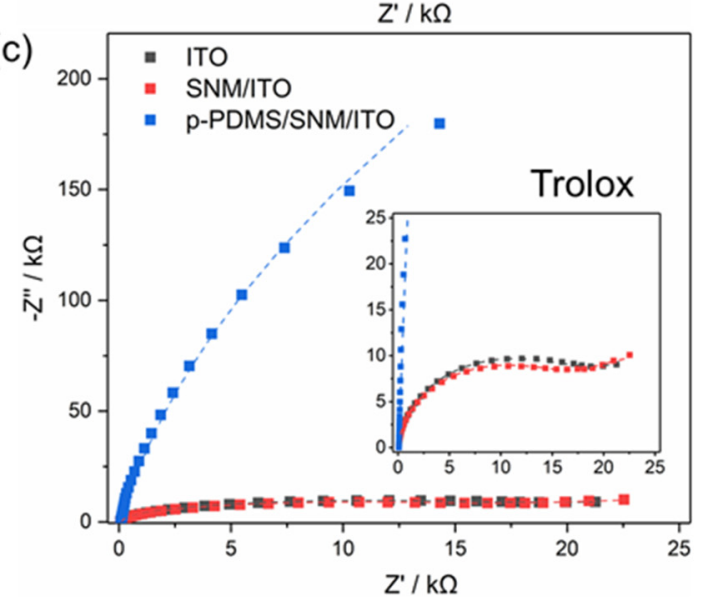

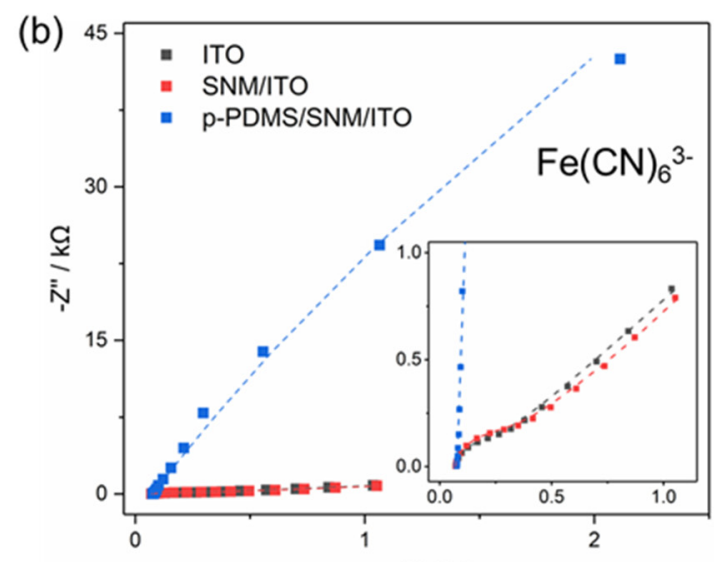

(d)

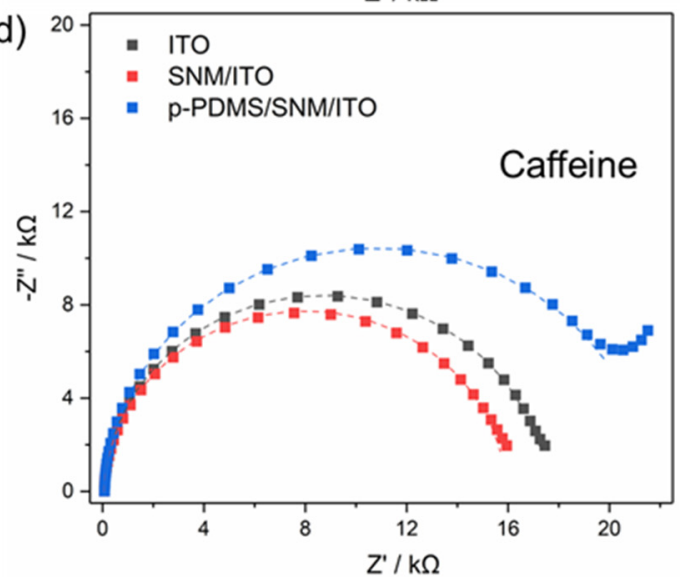

Fig. S4. Electrochemical impedance spectra (EIS) of $200 \mu \mathrm{M}$ (a) $\mathrm{Ru}\left(\mathrm{NH}_{3}\right)_{6}{ }^{3+}$, (b) $\mathrm{Fe}(\mathrm{CN})_{6}{ }^{3-}$, (c) Trolox and (d) caffeine in $0.5 \mathrm{M} \mathrm{KCl}$ (former three probes) or $0.2 \mathrm{M} \mathrm{K}_{2} \mathrm{SO}_{4}(\mathrm{pH}=1.5$, caffeine) recorded at the bare ITO (black), SNM/ITO (red) and p-PDMS/SNM/ITO (blue), frequency range $10^{4}-10^{-1} \mathrm{~Hz}$, signal amplitude of $10 \mathrm{mV}$.

(a)

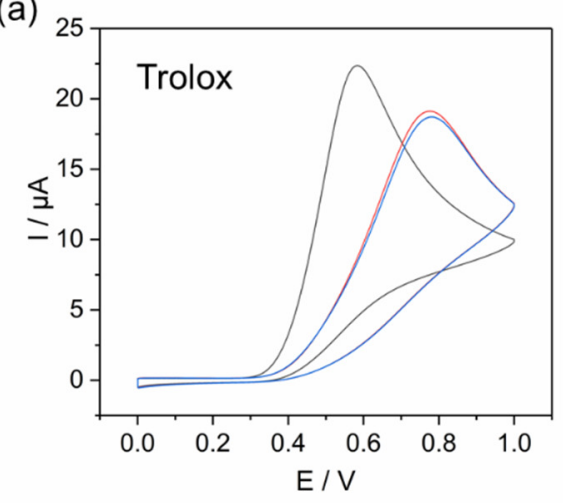

(b)

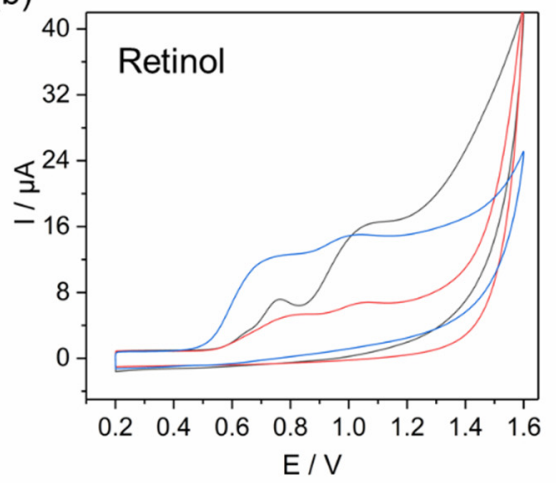

Fig. S5. Cyclic voltammograms of the bare ITO (black), SNM/ITO (red) and SNM/ITO modified with hydrophobic PDMS (blue) in $0.5 \mathrm{M} \mathrm{KCl}$ containing (a) Trolox and (b) retinol. The scan rate was $0.1 \mathrm{~V} / \mathrm{s}$. The hydrophobic PDMS modification was adopted the followed heating method ${ }^{4}$. The freshly prepared PDMS monolith was put on the top of the SNM/ITO with a distance of about 1 $\mathrm{mm}$ and heated on the hot plate at $100{ }^{\circ} \mathrm{C}$ for $10 \mathrm{~h}$. 


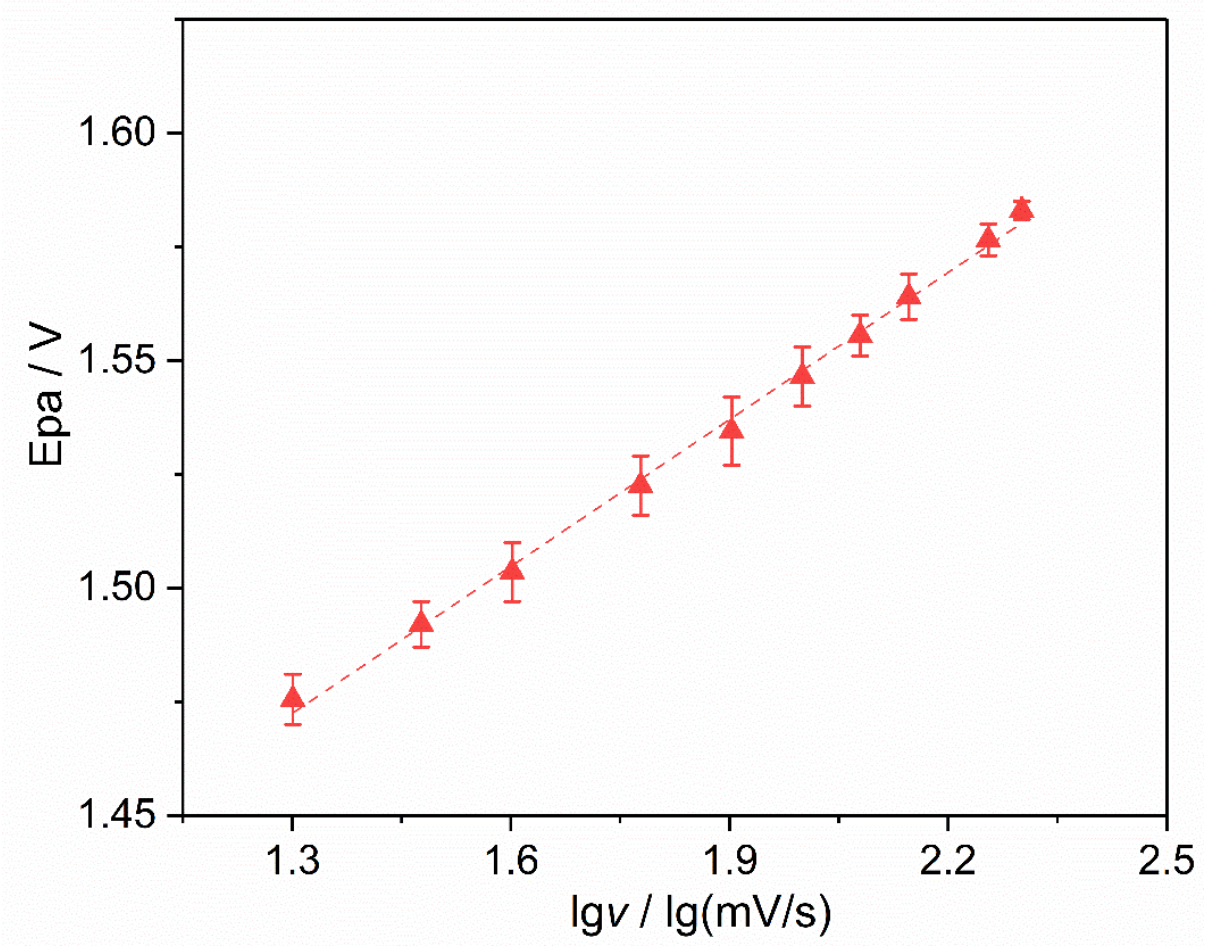

Fig. S6. The relationship between oxidation peak potentials of caffeine and scan rate. Based on Laviron's function, the following fitting equation is obtained. $\mathrm{E}_{\mathrm{pa}}=1.332+0.1077 \lg v\left(\mathrm{R}^{2}=\right.$ 0.9972).

4.Analytical condition optimization

(a)

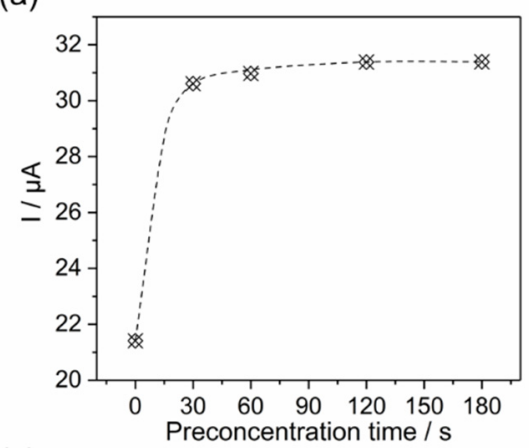

(c)

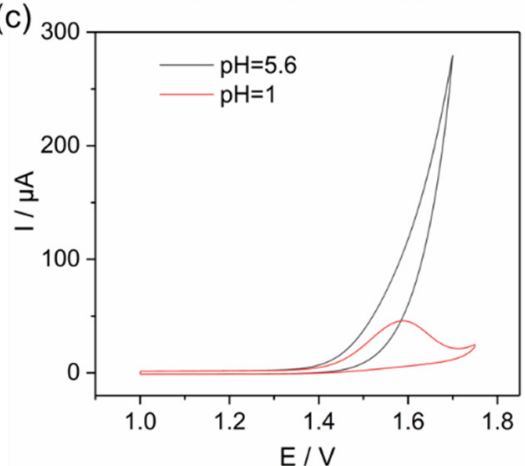

(b)

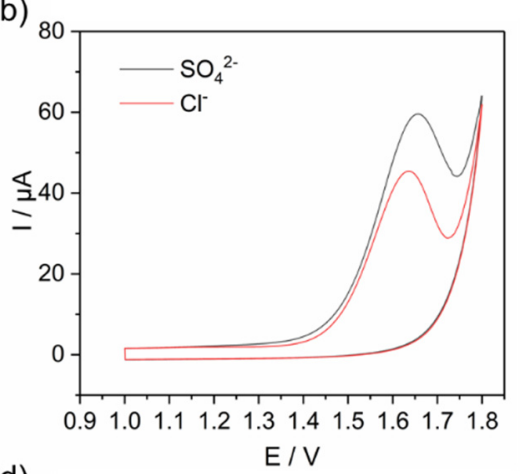

(d)

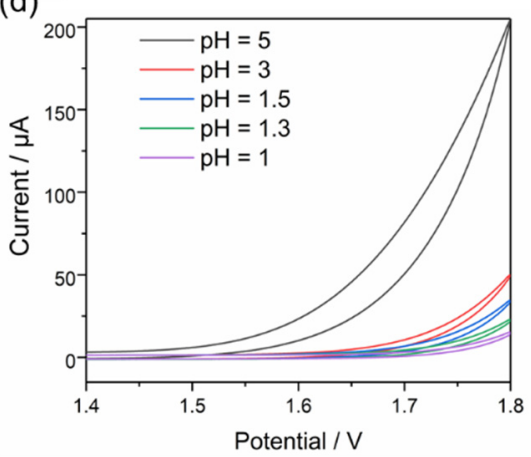


Fig. S7. (a) Effect of enrichment time on caffeine signal. (b) The voltammetry curve of caffeine with different electrolyte anions. (c, d) Cyclic voltammograms of p-PDMS/SNM/ITO in $0.5 \mathrm{M}$ $\mathrm{K}_{2} \mathrm{SO}_{4}$ under different $\mathrm{pH}$ in $200 \mu \mathrm{M}$ caffeine (c) and blank (d) solution. The scan rate was $0.1 \mathrm{v} / \mathrm{s}$.

5.Anti-interference and anti-fouling performance

(a)

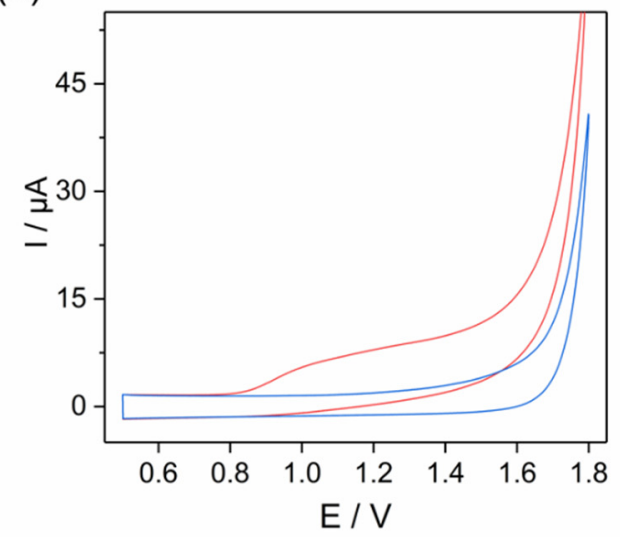

(b)

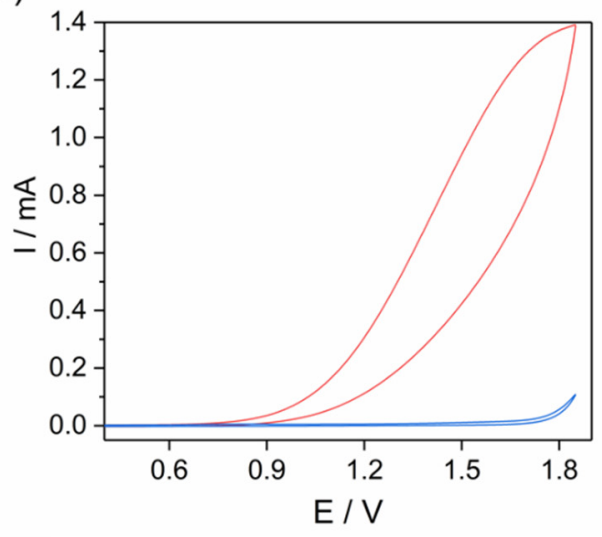

Fig. S8. (a) Cyclic voltammograms of the SNM/ITO (red) and PDMS/SNM/ITO (blue) in milk (a) and caffeine-free beverage (b).

\section{Comparison}

Table S1: Comparison of analytical performance of caffeine at p-PDMS/SNM/ITO with other modified electrodes in the literatures

\begin{tabular}{c|c|c|l|c}
\hline $\begin{array}{c}\text { Electrode type } \\
\text { configuration }\end{array}$ & Linear ranges & LODs & Analyzed interferences & Ref. \\
\hline $\begin{array}{c}\text { Fullerene/MWCNT/Nafion } \\
\text { /GCE }\end{array}$ & $10-1000 \mu \mathrm{M}$ & $72.9 \mathrm{nM}$ & Dopamine, $\mathrm{SO}_{3}^{2-}$ & 5 \\
\hline $\begin{array}{c}\text { Cerium Oxide Nanoparticle } \\
\text { Modified CPE }\end{array}$ & $5-80 \mu \mathrm{M}$ & $36 \mathrm{nM}$ & $\begin{array}{l}\mathrm{Na}^{+}, \mathrm{Ca}^{2+}, \mathrm{Ba}^{2+}, \mathrm{NO}_{3}{ }^{-}, \\
\text {sugar }\end{array}$ & 6 \\
\hline $\begin{array}{c}\text { MWCNT-AuNP-CPE } \\
\text { Boron doped diamond } \\
\text { electrode }\end{array}$ & $10-500 \mu \mathrm{M}$ & $354 \mathrm{nM}$ & $\begin{array}{l}\text { Uric acid, Theobromine, } \\
\text { Theophylline }\end{array}$ & 7 \\
\hline Zeolite/MWCNT modified & $1.70-104 \mu \mathrm{M}$ & $27 \mathrm{nM}$ & $\begin{array}{l}\text { Catechin, Gallic Acid, } \\
\text { Theobromine, } \\
\text { Theophylline }\end{array}$ & 8 \\
CPE & $10-500 \mu \mathrm{M}$ & $75 \mathrm{nM}$ & $\begin{array}{l}\text { Glucose, Sucrose, } \\
\text { Fructose, Citric Acid, } \\
\text { Ascorbic Acid, Nicotine, } \\
\text { Paracetamol, Theobromine, } \\
\text { Theophylline, Paraxanthine }\end{array}$ & 9 \\
\hline FC/Chi-MWCNTs/GPE & $\begin{array}{c}10-500 \mu \mathrm{M} \\
0.5-2.5 \mathrm{mM}\end{array}$ & $2.05 \mu \mathrm{M}$ & $\begin{array}{l}\text { Ascorbic acid, Dopamine, } \\
\text { Uric acid, Tryptophan, }\end{array}$ & 10 \\
\hline
\end{tabular}




\begin{tabular}{|c|c|c|c|c|}
\hline & & & Xanthine & \\
\hline $\begin{array}{l}\text { GO-reduced glutathione } \\
\text { modified CPE }\end{array}$ & $8-800 \mu \mathrm{M}$ & $153 \mathrm{nM}$ & $\begin{array}{l}\text { Glucose, Sucrose, } \\
\text { Fructose, Citric Acid, } \\
\text { Ascorbic Acid, Nicotine, } \\
\text { Paracetamol }\end{array}$ & 11 \\
\hline Pt- Graphene/GCE & $90-380 \mu \mathrm{M}$ & $112.9 \mathrm{nM}$ & $\begin{array}{l}\mathrm{Na}^{+}, \mathrm{K}^{+}, \mathrm{Cl}^{-}, \mathrm{NO}_{3}^{-}, \mathrm{Ca}^{2+}, \\
\text { Catechins, Glucose, } \\
\text { Tyrosine, Phenylalanine }\end{array}$ & 12 \\
\hline $\begin{array}{c}\text { GC-SnS/TiO2@GO coated } \\
\text { GCE }\end{array}$ & $\begin{array}{c}16.6 \mathrm{nM}-333 \\
\mathrm{nM}\end{array}$ & $4.4 \mathrm{nM}$ & $\begin{array}{l}\mathrm{NaCl}, \mathrm{NaAc}, \mathrm{KCl} \text {, Uric } \\
\text { acid, Histidine }\end{array}$ & 13 \\
\hline $\begin{array}{c}\text { (Nafion/GNPs) })_{\mathrm{n}} \text { modified } \\
\text { GCE }\end{array}$ & $50-5000 \mu \mathrm{M}$ & $24 \mu \mathrm{M}$ & Not mentioned & 14 \\
\hline $\begin{array}{c}\text { Cassava Starch- } \mathrm{Fe}_{3} \mathrm{O}_{4} \\
\text { Modified GCE }\end{array}$ & $50-900 \mu \mathrm{M}$ & $23 \mu \mathrm{M}$ & Not mentioned & 15 \\
\hline Nafion/GCE & $63.1-600 \mu \mathrm{M}$ & $18.6 \mu \mathrm{M}$ & $\begin{array}{l}\text { Taurine, Calcium } \\
\text { Pantothenate, Niacinamide, } \\
\text { Ascorbic Acid }\end{array}$ & 16 \\
\hline $\begin{array}{c}\text { Attapulgite/nafion coated } \\
\text { GCE }\end{array}$ & $0.2-4.4 \mu \mathrm{M}$ & $226 \mathrm{nM}$ & $\begin{array}{l}\text { Citric acid, Glucose, } \\
\text { Dopamine, } \mathrm{SO}_{3}{ }^{2-}\end{array}$ & 17 \\
\hline PDMS/SNM/ITO & $0.05-700 \mu \mathrm{M}$ & $20 \mathrm{nM}$ & $\begin{array}{l}\text { Trolox, Ascorbic acid, } \\
\text { Theobromine, } \\
\text { Theophylline, Xanthine, } \\
\text { Hypoxanthine, Purine, L- } \\
\text { Tyrosine, Tea polyphenols }\end{array}$ & $\begin{array}{l}\text { This } \\
\text { work }\end{array}$ \\
\hline
\end{tabular}

MWCNT: Multi-walled Carbon Nanotubes; GCE: Glassy Carbon Electrode; CPE: Carbon Paste Electrode; GO: Graphene oxide; FC: Ferrocene; Chi: Chitosan; GNP: Graphite Nanoplatelets

\section{References}

1. Mazaltarim, A. J.; Taylor, J. M.; Konda, A.; Stoller, M. A.; Morin, S. A., Mechanically Induced Hydrophobic Recovery of Poly(dimethylsiloxane) (PDMS) for the Generation of Surfaces with Patterned Wettability. ACS Appl. Mater. Interfaces 2019, 11 (36), 33452-33457.

2. Kinney, C. R., A System Correlating Molecular Structure of Organic Compounds with their Boiling Points. I. Aliphatic Boiling Point Numbers. J. Am. Chem. Soc. 1938, 60 (12), 3032-3039.

3. Bodas, D.; Khan-Malek, C., Formation of more stable hydrophilic surfaces of PDMS by plasma and chemical treatments. Microelectron. Eng. 2006, 83 (4), 1277-1279.

4. Yuan, J.; Liu, X.; Akbulut, O.; Hu, J.; Suib, S. L.; Kong, J.; Stellacci, F., Superwetting nanowire membranes for selective absorption. Nat. Nanotechnol. 2008, 3 (6), 332-6.

5. Tajeu, K. Y.; Dongmo, L. M.; Tonle, I. K., Fullerene/MWCNT/Nafion Modified Glassy Carbon Electrode for the Electrochemical Determination of Caffeine. American Journal of Analytical Chemistry 2020, 11 (2), 114 127.

6. Santhosh, B. M.; Manjunatha, S.; Shivakumar, M.; Dharmaprakash, M. S.; Manjappa, S., Electrochemical Investigation of Caffeine by Cerium Oxide Nanoparticle Modified Carbon Paste Electrode. Journal of the Electrochemical Society 2020, 167 (4), 047503. 
7. Ferrag, C.; Noroozifar, M.; Kerman, K., Thiol functionalized carbon ceramic electrode modified with multi-walled carbon nanotubes and gold nanoparticles for simultaneous determination of purine derivatives. Mater Sci Eng C Mater Biol Appl 2020, 110, 110568.

8. Júnior, P. C. G.; dos Santos, V. B.; Lopes, A. S.; de Souza, J. P. I.; Pina, J. R. S.; Júnior, G. C. A. C.; Marinho, P. S. B., Determination of theobromine and caffeine in fermented and unfermented Amazonian cocoa (Theobroma cacao L.) beans using square wave voltammetry after chromatographic separation. Food Control 2020, 108, 106887.

9. Azab, S. M.; Shehata, M.; Fekry, A. M., A novel electrochemical analysis of the legal psychoactive drug caffeine using a zeolite/MWCNT modified carbon paste sensor. New Journal of Chemistry 2019, 43 (38), 15359-15367.

10. Li, S. P.; Noroozifar, M.; Kerman, K., Nanocomposite of ferricyanide-doped chitosan with multi-walled carbon nanotubes for simultaneous senary detection of updates redox-active biomolecules. Journal of Electroanalytical Chemistry 2019, 849, 113376.

11. Shehata, M.; Azab, S.; Fekry, A., May glutathione and graphene oxide enhance the electrochemical detection of caffeine on carbon paste sensor in aqueous and surfactant media for beverages analysis? Synth. Met. 2019, 256, 116122.

12. Qiao, J.; Zhang, L.; Gao, S.; Li, N., Facile Fabrication of Graphene-Supported Pt Electrochemical Sensor for Determination of Caffeine. Appl Biochem Biotechnol 2020, 190 (2), 529-539.

13. Murugan, E.; Kumar, K., Fabrication of SnS/TiO2@GO Composite Coated Glassy Carbon Electrode for Concomitant Determination of Paracetamol, Tryptophan, and Caffeine in Pharmaceutical Formulations. Analytical Chemistry 2019, 91 (9), 5667-5676.

14. Hernandez-Aldave, S.; Tarat, A.; McGettrick, J. D.; Bertoncello, P., Voltammetric Detection of Caffeine in Beverages at Nafion/Graphite Nanoplatelets Layer-by-Layer Films. Nanomaterials 2019, 9 (2), 221.

15. Mulyasuryani, A.; Tjahjanto, R. T.; Andawiyah, R. a., Simultaneous Voltammetric Detection of Acetaminophen and Caffeine Base on Cassava Starch-Fe304 Nanoparticles Modified Glassy Carbon Electrode. Chemosensors 2019, 7 (4), 49.

16. Farag, A. S.; Pravcová, K.; C Česlová, L.; Vytřas, K.; Sýs, M., Simultaneous determination of caffeine and pyridoxine in energy drinks using differential pulse voltammetry at glassy carbon electrode modified with Nafion®. Electroanalysis 2019, 31 (8), 1494-1499.

17. Thu, P. T. K.; Trinh, N. D.; Hoan, N. T. V.; Du, D. X.; Mau, T. X.; Trung, V. H.; Phong, N. H.; Toan, T. T. T.; Khieu, D. Q., Synthesis of cobalt ferrite and simultaneous determination of ascorbic acid, acetaminophen and caffeine by voltammetric method using cobalt ferrite modified electrode. Journal of Materials Science-Materials in Electronics 2019, 30 (18), 17245-17261. 